Bull. Austral. Math. Soc.

VOL. 38 (1988) $[317-319]$

\title{
A STOCHASTIC ANALYSIS OF SCORING SYSTEMS
}

\section{Graham Hilford Pollard}

Many scoring systems can be seen as statistical tests of hypotheses [1]. In tennis singles, for example, the scoring system can be seen as a test involving 2 binomial probabilities $p_{a}$ and $p_{b}$ where $p_{a}\left(p_{b}\right)$ is the probability player $A$ (player $B$ ) wins a point initiated by player $A$ (player $B$ ). Tennis singles is thus a bipoints game. The tennis scoring system is an inefficient test relative to the sequential probability ratio test ( $S P R T$ ) based on pairs of these points. Miles showed that when $p_{a}+p_{b}>1$ (the tennis context), an SPRT based on the play-the-loser ( $P L)$ rule is super-efficient. Chapter 2 of this thesis shows that, when $p_{a}+p_{b}>1$, there is in fact a spectrum of super-efficient tests (with even durations) based on partial-PL (PPL) rules. The most efficient tests within this spectrum, when $p_{a}+p_{b}>1$, are the $S P R T$ based on the (full) $P L$ rule. Chapter 3 extends this spectrum of tests to produce the total spectrum of tests (including those with odd durations).

Points within the tennis scoring system have different importances [2] whereas points within any member of the above (efficient) spectrum of $P P L$ systems are seen to be equally important when $p_{a}=p_{b}$. Intuitively, the differing importances of the points within the tennis scoring system contribute to the inefficiency of that system. Chapter 4 establishes a relationship between the efficiency of a bipoints scoring system and the importances of the points within it; a relationship which is used in Chapter 5 to show that the $S P R T$ based on the $P L$ (play-the-winner, $P W$ ) rule has an optimal efficiency property when $p_{a}+p_{b}>1\left(p_{a}+p_{b}<1\right)$. Thus Chapter 5 solves a well-known 2-sample binomial problem.

Chapter 6 shows that some complex SPRT systems can be decomposed into smaller independent components called modules which can in turn be analysed to produce values from which the asymptotic efficiency of the complete $S P R T$ system can be evaluated. This module approach is used to give an intuitive explanation as to why the $P L$ rule is more (less) efficient than the $P W$ rule when $p_{a}+p_{b}>1\left(p_{a}+p_{b}<1\right)$. In another example, the module which produces the asymptotically most efficient $S P R T$ for the case in which $\alpha$, the probability of a type I error and $\beta$, the probability of a

Received 11 December 1988. Thesis submitted to Australian National University, December 1986. Degree approved December 1987. Supervisor Dr. Roger Miles.

Copyright Clearance Centre, Inc. Serial-fee code: 0004-9729/88 \$A2.00+0.00. 
type II error are equal ( $\alpha=\beta$ is required for the fairness of a scoring system) is also the asymptotically most efficient for the case in which $\alpha \neq \beta$.

Chapter 7 uses the module approach to show that the super-efficiency of the $P L$ rule carries over to the case of tennis doubles in which there are essentially 4 binomial probabilities $p_{a 1}, p_{a 2}, p_{b 1}$ and $p_{b 2}$ (provided $\bar{p}_{a}+\bar{p}_{b}>1$ and $\left|p_{a 1}-p_{a 2}\right|=\left|p_{b 1}-p_{b 2}\right|$ ).

The particular scoring system currently used in tennis is analysed in Chapter 8 (see also [3]) and the methodology used is seen to be useful for analysing any nested scoring system (for example, tennis is 3-nested: points - games - sets). It was the study of this specific scoring system and its inherent inefficiency which lead to the theory of Chapters 2 to 7 . A new tennis scoring system with a smaller variance of duration is proposed in Chapter 8. (See also [5].)

Chapter 9 contains a brief discussion of some of the characteristics that need to be considered by the designer of a scoring system. In particular, the roles played by the expected duration, the variance of duration and the efficiency of a system are discussed.

In Chapter 10 an exact relationship between the increased probability of winning a point or set of points and the increase in the probability of winning a match is given. An alternative approach shows that points or states can interact positively or negatively.

In Chapter 11 team play with associated countback rules is investigated. The general conclusion is that upward-nested countback systems (for example points - games - sets, in tennis) are preferable to downward-nested ones (sets - games - points).

In Chapter 12 it is shown that the classical scoring system used in multiple choice examinations can be considerably improved by modifying that scoring system and instructing the examinees to cross any boxes known to be incorrect when the correct box for that question is unknown (see also [4]). A scoring system which, it is argued, should remove random guessing completely is also given. (See also [6].)

\section{REFERENCES}

[1] R.E. Miles, 'Symmetric sequential analysis: the efficiiencies of sports scoring systems (with particular reference to those of tennis)', J.R. Statist. Soc. B 46(1) (1984), 93-108.

[2] C:. Morris, 'The most important points in tennis', in Optimal Strategies in Sport: Vol. 5 in Studies in management science and systems, Edited by S.P. Ladany and R.E. Machol, p. 131-140 (North Holland, Amsterdam, 1977).

[3] G.H. Pollard, 'An analysis of classical and tie-breaker tennis', Austral. J. Statist. 25(3) (1983), 496-505.

[4] G.H. Pollard, 'Scoring in multiple choice examinations', Math. Scientist 10 (1985), 93-97.

[5] G.H. Pollard, 'A new tennis scoring system', Research Quarterly for Exercise and Sport 58 (1987), 229-233.

[6] G.H. Pollard, 'Scoring to remove guessing in multiple choice examinations', Internat. J. Math. Ed. Sci. Tech. (to appear). 


\section{School of Information Sciences}

Camberra College of Advanced Education

P.O. Box 1

Belconnon, A.C.T. 2616

Australia 